\title{
Gamma-ray burst detection with the AGILE mini-calorimeter
}

\author{
M. Marisaldi ${ }^{1}$, C. Labanti $^{1}$, F. Fuschino ${ }^{1}$, M. Galli ${ }^{2}$, A. Argan ${ }^{3}$, G. Barbiellini ${ }^{4,5}$, M. Basset ${ }^{5}$, F. Boffelli ${ }^{6,7}$,

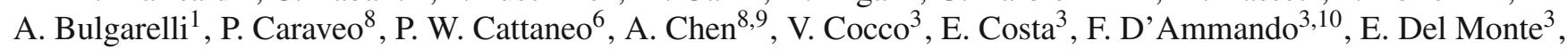

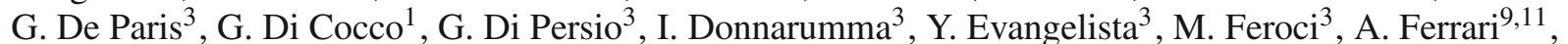 \\ M. Fiorini ${ }^{8}$, L. Foggetta ${ }^{12,13}$, T. Froysland ${ }^{9,10}$, M. Frutti ${ }^{3}$, F. Gianotti ${ }^{1}$, A. Giuliani ${ }^{8}$, I. Lapshov ${ }^{3,14}$, F. Lazzarotto ${ }^{3}$, \\ F. Liello ${ }^{4,5}$, P. Lipari ${ }^{15,16}$, F. Longo ${ }^{4,5}$, M. Mastropietro ${ }^{17}$, E. Mattaini ${ }^{8}$, A. Mauri ${ }^{1}$, F. Mauri ${ }^{6}$, S. Mereghetti ${ }^{8}$, \\ E. Morelli ${ }^{1}$, A. Morselli ${ }^{18}$, L. Pacciani ${ }^{3}$, A. Pellizzoni ${ }^{8}$, F. Perotti ${ }^{8}$, P. Picozza ${ }^{18}$, C. Pontoni ${ }^{5}$, G. Porrovecchio ${ }^{3}$, \\ M. Prest ${ }^{12,13}$, G. Pucella ${ }^{3}$, M. Rapisarda ${ }^{19}$, A. Rappoldi ${ }^{6}$, E. Rossi ${ }^{1}$, A. Rubini ${ }^{3}$, P. Soffitta $^{3}$, M. Tavani $^{3,10}$, \\ A. Traci ${ }^{1}$, M. Trifoglio ${ }^{1}$, A. Trois ${ }^{3}$, E. Vallazza ${ }^{5}$, S. Vercellone ${ }^{8}$, V. Vittorini ${ }^{10}$, A. Zambra ${ }^{8,9}$, D. Zanello ${ }^{15,16}$, \\ C. Pittori ${ }^{20}$, F. Verrecchia ${ }^{20}$, S. Cutini ${ }^{20}$, D. Gasparrini ${ }^{20}$, B. Preger ${ }^{20}$, P. Santolamazza ${ }^{20}$, P. Giommi ${ }^{20}$, \\ L. A. Antonelli ${ }^{20}$, S. Colafrancesco ${ }^{20}$, and L. Salotti ${ }^{21}$
}

(Affiliations can be found after the references)

Received 10 July 2008 / Accepted 13 August 2008

\begin{abstract}
Context. The mini-calorimeter (MCAL) instrument on-board the AGILE satellite is a non-imaging gamma-ray scintillation detector sensitive in the $300 \mathrm{keV}-100 \mathrm{MeV}$ energy range with a total on-axis geometrical area of $1400 \mathrm{~cm}^{2}$. Gamma-ray bursts (GRBs) are one of the main scientific targets of the AGILE mission and the MCAL design as an independent self-triggering detector makes it a valuable all-sky monitor for GRBs. Furthermore MCAL is one of the very few operative instruments with microsecond timing capabilities in the MeV range.

Aims. In this paper the results of GRB detections with MCAL after one year of operation in space are presented and discussed.

Methods. A flexible trigger logic implemented in the AGILE payload data-handling unit allows the on-board detection of GRBs. For triggered events, energy and timing information are sent to telemetry on a photon-by-photon basis, so that energy and time binning are limited by counting statistics only. When the trigger logic is not active, GRBs can be detected offline in ratemeter data, although with worse energy and time resolution. Results. Between the end of June 2007 and June 2008 MCAL detected 51 GRBs, with a detection rate of about 1 GRB/week, plus several other events at a few milliseconds timescales. Since February 2008 the on-board trigger logic has been fully active. Comparison of MCAL detected events and data provided by other space instruments confirms the sensitivity and effective area estimations. MCAL also joined the 3rd Inter-Planetary Network, to contribute to GRB localization by means of triangulation.
\end{abstract}

Key words. gamma rays: bursts - instrumentation: detectors

\section{Introduction}

The AGILE satellite (Tavani et al. 2008b,a), the Italian space mission dedicated to gamma-ray and hard- $X$ astrophysics, has the study of GRBs among its main scientific targets. The gammaray imaging detector (GRID), composed of a tungsten-silicon tracker (Prest et al. 2003) and a CsI(Tl) Mini-Calorimeter, has a wide field of view that makes it a valuable instrument for GRB detection in the poorly explored $30 \mathrm{MeV}-50 \mathrm{GeV}$ energy band. SuperAGILE (Feroci et al. 2007b), the hard X-ray imager on-board AGILE operating in the $18-60 \mathrm{keV}$ energy band, is equipped with an on-board trigger logic and localization algorithm providing few arcmin position accuracy, allowing rapid dissemination of the coordinates (Del Monte et al. 2007). The Mini-Calorimeter, despite being a subsystem of the GRID, is also equipped with a self-triggering operative mode and on-board logic making it an all-sky monitor in the $300 \mathrm{keV}$ $100 \mathrm{MeV}$ energy range. A simultaneous GRB detection with GRID, MCAL and SuperAGILE would allow spectral coverage over six orders of magnitude. In this paper the status of the GRB detection with MCAL, one year after the AGILE launch, is reviewed and discussed.

\section{MCAL GRB detection capabilities}

MCAL is composed of $30 \mathrm{CsI}(\mathrm{Tl})$ scintillator bars (dimensions: $15 \times 23 \times 375 \mathrm{~mm}^{3}$ each) arranged in two orthogonal layers, for a total thickness of 1.5 radiation lengths. In a bar the readout of the scintillation light is accomplished by two custom PIN Photodiodes (PD) coupled one at each short side of the bar. Detailed descriptions of MCAL can be found in Labanti et al. (2006, 2008).

MCAL works in two possible operative modes:

- in GRID mode a trigger issued by the silicon tracker starts the collection of all the detector signals in order to determine the energy and position of particles converted in the tracker and interacting in MCAL;

- in BURST mode each bar behaves as an independent self triggering detector and generates a continuous stream of gamma-ray events in the energy range $300 \mathrm{keV}-100 \mathrm{MeV}$. The dynamic range of the electronics was set so high to account also for the high energy emission from GRBs. Nevertheless, due to the limited thickness of the detector, secondaries originated by interactions of photons at energies higher than about $10 \mathrm{MeV}$ are expected to give rise to 
significant incomplete signal collection, making the energy reconstruction of the incident photon more difficult.

Both operative modes can be active at the same time, but the one relevant to GRB investigation is the BURST mode. BURST data are stored in a circular buffer and analysed by a dedicated trigger logic, described in detail in Fuschino et al. (2008a). If a trigger is issued, the data are sent to telemetry on a photon-byphoton basis including, for each event, energy information and a time tag with $2 \mu$ s accuracy. Without a trigger, or when the trigger logic is off, due to telemetry limitations BURST data are not sent to the ground on a photon-by-photon basis, but are used to build two broad band energy spectra (Scientific Ratemeters, SRM), one for each detection layer, and stored in telemetry with a $1.024 \mathrm{~s}$ time bin.

Due to programmatic constraints it was not possible to switch on and configure the on-board trigger logic prior to the end of November 2007. Then it was switched off again during January 2008, and since the 5th of February 2008 it has again been operative. When the trigger logic was not active, GRBs were detected by on-ground analysis, scanning the SRM data for rate increases with a dedicated software task. Despite several GRBs having been detected with this method, the coarse time and energy binning limits the scientific exploitation of the data. On the contrary, with the onset of the on-board trigger logic, time and energy binning for triggered events is only limited by counting statistics. The early MCAL GRB detections are reported in Marisaldi et al. (2008).

Several GRB detectors are currently active in space, each with its own specific characteristics. Apart from Swift-BAT (Barthelmy et al. 2000), INTEGRAL-IBIS (Ubertini et al. 2003), SuperAGILE (Feroci et al. 2007b), GLAST-GBM (Meegan et al. 2007) and GLAST-LAT (Michelson 2007), all the other detectors have no or very limited imaging capabilities and rely on triangulation between different spacecraft for GRB localization, through the 3rd inter-planetary network (IPN) ${ }^{1}$. Among the current IPN instruments, only three have spectroscopic capabilities at $\mathrm{MeV}$ energies, in an energy range partially overlapping with that of MCAL: Konus-Wind (Aptekar et al. 1995), Suzaku-WAM (Yamaoka et al. 2006) and RHESSI (Wigger et al. 2004). Among these, only the RHESSI spectrometer is capable of photon-by-photon data download. Also GLAST-GBM, expected to join the IPN too, has both spectral capabilities in the $\mathrm{MeV}$ range and photon-by-photon data download for triggered events. GLAST-LAT has both high spectral and timing capabilities, but in an energy range higher than that of MCAL.

\section{Results}

\subsection{GRB detections}

Between 22nd June 2007 and 30th June 2008 MCAL detected 51 GRBs, with an average detection rate of about $1 \mathrm{GRB} /$ week. Most of these detections have been independently confirmed by other instruments. Only 16 events have been localized, either by Swift, SuperAGILE or the IPN, as reported in Table 1. The IPN localizations reported here are those publicly available at the time of writing; since most of the MCAL events have also been detected by other IPN instruments (Hurley 2008) the number of IPN localizations is expected to rise when the IPN catalogues become available. The detection rate is in good agreement with the sensitivity estimations reported in Ghirlanda et al. (2004). It

${ }^{1}$ IPN web page: http://www.ssl. berkeley.edu/ipn3/
Table 1. MCAL GRB detection summary.

\begin{tabular}{lr}
\hline \hline MCAL detection & Number of events \\
\hline Ground trigger $^{a}$ & 28 \\
On-board trigger & 23 \\
Localized by SuperAGILE & 1 \\
Localized by Swift & 10 \\
Localized by IPN $^{c}$ & 5 \\
\hline
\end{tabular}

${ }^{a}$ Scientific ratemeters only: 22nd Jun. 2007-24th Nov. 2007, 1st Jan. 2008-4th Feb. 2008.

${ }^{b}$ 25th Nov. 2007-31st Dec. 2007, 5th Feb. 2008-30th Jun. 2008.

${ }^{c}$ Public coordinates available in GCN.

must also be noted that in the same time period SuperAGILE localized another four GRBs that were not detected by MCAL.

Figure 1 shows the MCAL light curves for a sample of GRBs. Panels (a), (b) and (c) refer to GRBs triggered on the ground based on SRM data. For these events the on-board trigger logic was not active; only the light curves relative to the upper detection layer (the one closer to the silicon tracker) are shown. Panels (d) to (k) refer to GRBs triggered on-board; the light curves relative to the complete instrument are shown. Panels (d) to (f) refer to $<5 \mathrm{~s}$ long GRBs, shown here with a $32 \mathrm{~ms}$ time bin. Panels ( $\mathrm{g}$ ) to $(\mathrm{k})$ refer to longer GRBs, shown here with a $256 \mathrm{~ms}$ time bin. For those events with a public localization available the GRB name is reported too.

Figure 2 is a polar plot centered at the pointing direction of the AGILE satellite, showing the position of the localized GRBs detected by MCAL in the considered period. Coordinates for GRB 070915 and GRB 070825, provided by IPN but not published in GCN, are also included (Pal'shin \& Hurley 2007). Several GRBs have been detected at off-axis angles greater than $90^{\circ}$, with the highest being the bright GRB 071020, localized by Swift (Holland et al. 2007), detected at $166^{\circ}$ off-axis, i.e. coming almost from a direction opposite to the AGILE pointing. Despite that for $>90^{\circ}$ events it is difficult to provide reliable spectral information, due to the still incomplete modeling of the spacecraft shell with Monte Carlo simulations (Longo et al. 2002; Cocco et al. 2002), it demonstrates the MCAL all-sky detection capabilities.

The only GRB detected by the GRID detector above $50 \mathrm{MeV}$ in the considered period is GRB 080514B (Rapisarda et al. 2008; Giuliani et al. 2008b), which also triggered MCAL and SuperAGILE and is the subject of a dedicated paper (Giuliani et al. 2008a). This GRB is further considered in Sect. 3.3 regarding spectral fitting of MCAL data.

\subsection{Sensitivity}

Figure 3 shows the MCAL sensitivity to GRBs. The sensitivity has been calculated according to the procedure described in Band (2003) and reports the peak flux in the 100-1000 keV energy band at the detector's threshold ( $5 \sigma$ significance above background in $1 \mathrm{~s}$ ), as a function of the peak energy, for three sample spectral shapes modeled according to the Band Model (Band et al. 1993). The sensitivity curves have been calculated using the MCAL efficiency at a $40^{\circ}$ off-axis angle resulting from Monte Carlo simulations and the in-flight background level (about 210 counts/s over all the detector in the 330-700 keV trigger band). Superimposed on the sensitivity curves are data points corresponding to GRBs with public spectral parameters, published as GRB Coordinates Network circulars either by the Suzaku-WAM or Konus-Wind teams. Solid triangles refer to 

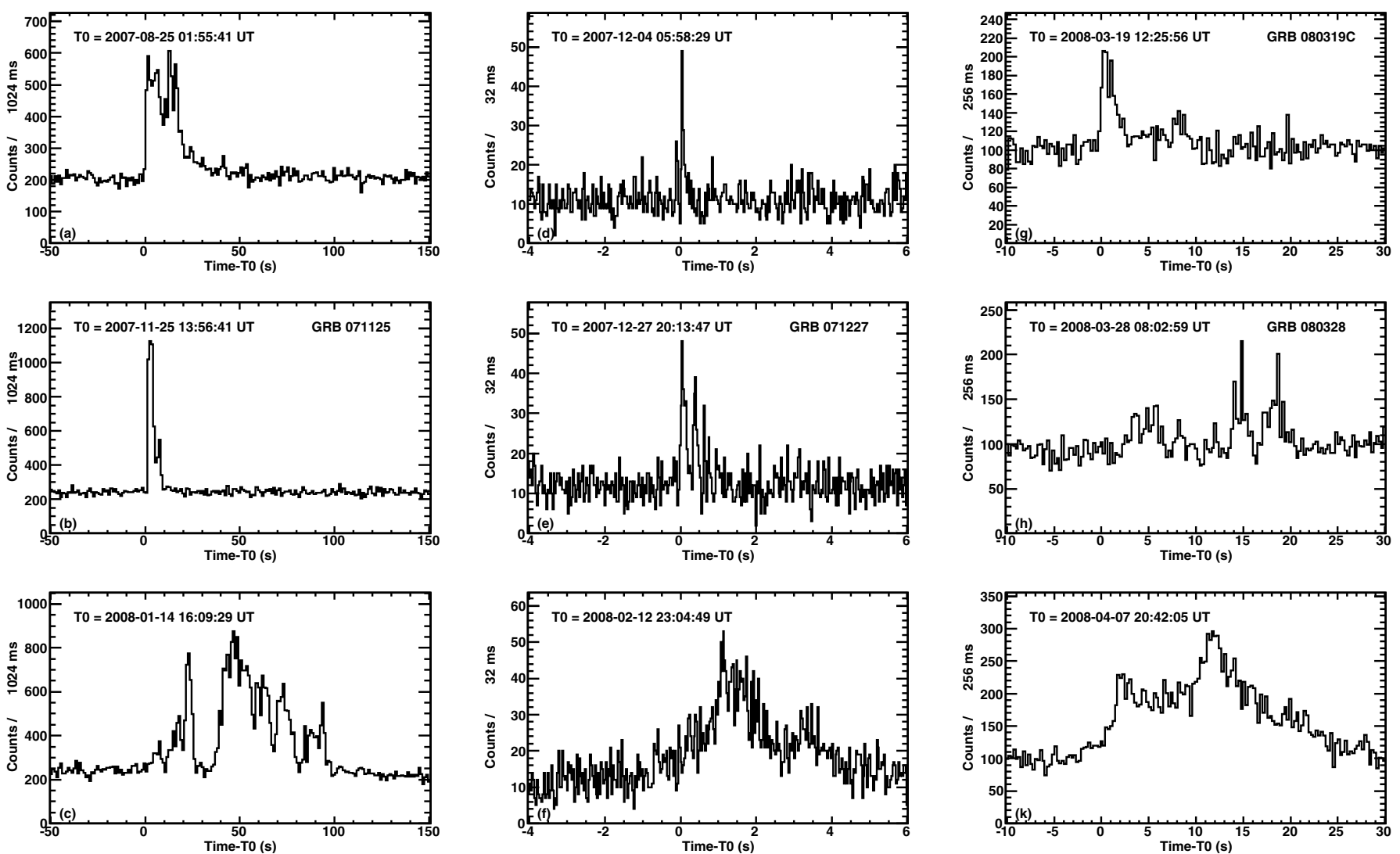

Fig. 1. Light curves of several bursts detected by MCAL. Panels a) to c): GRBs triggered on the ground by SRM data. Panels d) to k): GRBs triggered by the on-board logic.

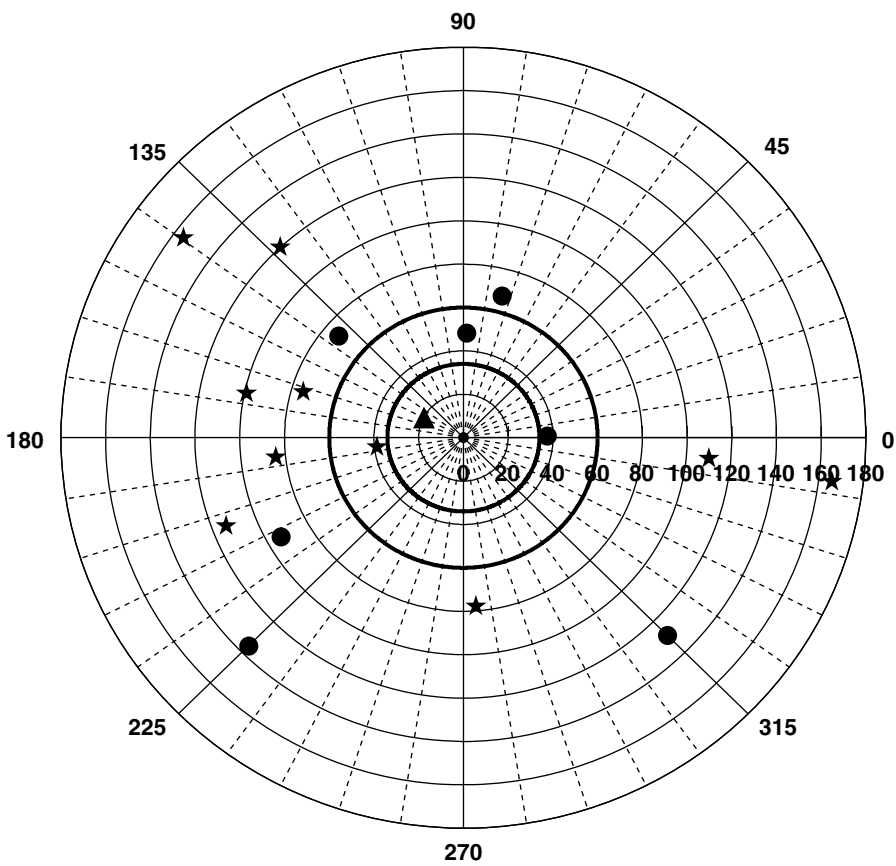

Fig. 2. Positions of the GRBs detected by MCAL and localized either by Swift (stars), IPN (circles), or SuperAGILE (triangle) in the reference frame of AGILE pointing. The radial coordinate is the off-axis angle with respect to the pointing of the satellite. The thick solid circles represent the GRID and SuperAGILE (the innermost) fields of view.

GRBs also detected by MCAL at an incident angle lower than 90: GRB 071125 (Ohno et al. 2007; Golenetskii et al. 2007a),

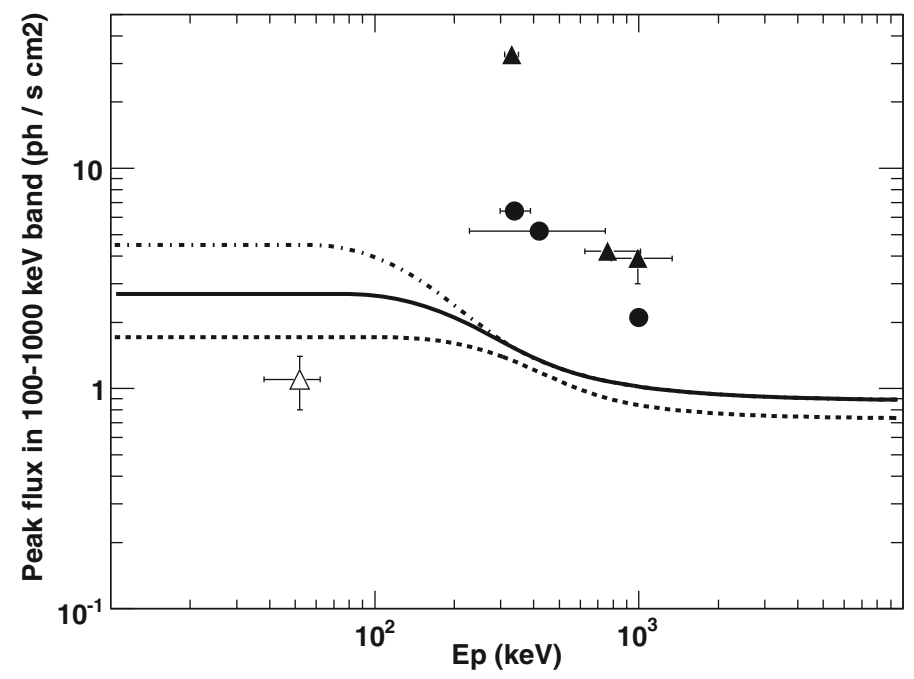

Fig. 3. MCAL sensitivity as a function of the peak energy for $40^{\circ}$ off axis GRBs with different Band spectral models. Continuous line: $\alpha=-1, \beta=-2.5$; dashed line $\alpha=-0.5, \beta=-2$; dot-dashed line $\alpha=-1, \beta=-3$. Solid triangles: GRBs detected by MCAL at incident angle $<90^{\circ}$. Solid circles: GRBs detected by MCAL at incident angle $>90^{\circ}$. Hollow triangle: GRB at incident angle $<90^{\circ}$ but not detected by MCAL.

GRB 080204 (Terada et al. 2008; Golenetskii et al. 2008b), GRB 080319C (Onda et al. 2008b; Golenetskii et al. 2008c). Solid circles refer to GRBs detected by MCAL at incident angles greater than 90 : GRB 071227 (Onda et al. 2008a; 


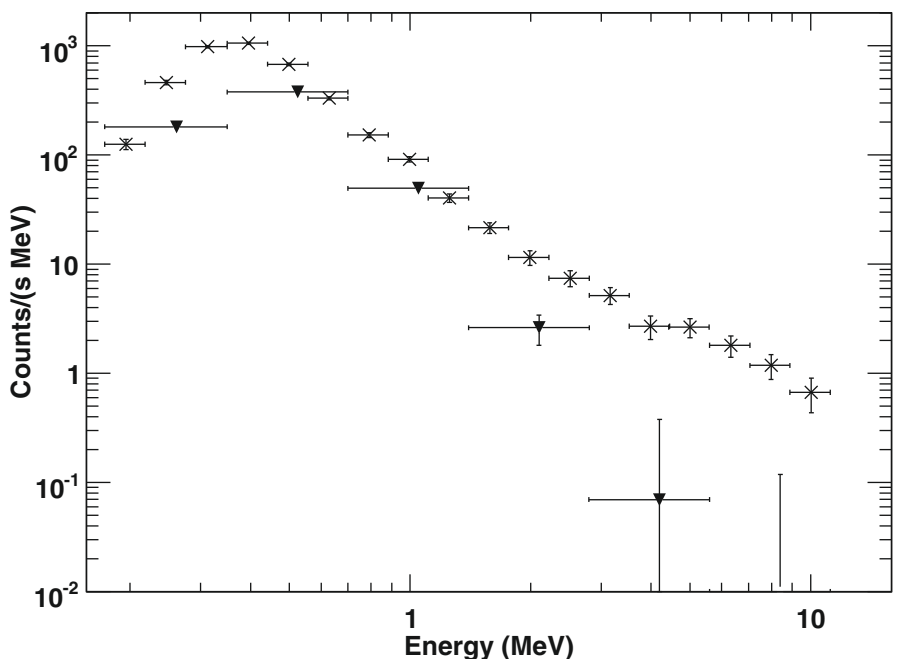

Fig. 4. MCAL count spectra for GRB 080407B. Filled triangles: upper detection layer SRM count spectrum. Crosses: count spectrum obtained from photon-by-photon data relative to the whole MCAL.

Golenetskii et al. 2007d), GRB 080122 (Uehara et al. 2008; Golenetskii et al. 2008a), GRB 080328 (Kodaka et al. 2008; Golenetskii et al. 2008d). For this plot, the Suzaku-WAM peak flux values have been used because they have been homogeneously provided for the $100 \mathrm{keV}-1 \mathrm{MeV}$ band.

The hollow triangle point below the sensitivity curves corresponds to GRB 071010B, localized by Swift and observed at high energy by Suzaku-WAM and Konus-Wind (Kira et al. 2007c; Golenetskii et al. 2007c). This GRB was not detected by MCAL, despite it being unocculted by the Earth and its incoming direction was just $41^{\circ}$ off-axis, as expected from its spectral parameters and the MCAL sensitivity curve. Other GRBs in similar conditions are GRB 070704 (Kira et al. 2007b,a) and GRB 070724B (Feroci et al. 2007a; Endo et al. 2007; Golenetskii et al. 2007b). GRB 070724B has been localized by SuperAGILE and was classified as "No High Energy" (Del Monte et al. 2008).

\subsection{Energy calibration}

One of the main characteristics of MCAL is the broad energy range extending up to several $\mathrm{MeV}$. To take full advantage of the extended spectral coverage, operation with the on-board logic active is mandatory, as SRMs provide only a coarse spectral resolution. Figure 4 shows two count spectra for GRB 080407 (trigger time 2008-04-07 20:42:05 UT), the highest fluence GRB triggered on-board by MCAL in the considered period. One spectrum has been obtained from SRM data relative to the upper detection layer (filled triangles), the other is obtained from photon-by-photon data (crosses). Photon-by-photon data obviously allow a much finer spectral reconstruction. Moreover these data are detected in the whole MCAL, while SRM spectra are obtained separately for the two detection layers, making the energy reconstruction more difficult at energies above a few $\mathrm{MeV}$ where Compton scattering becomes more important and photons tend to produce multiple hits on different detection layers. The total number of GRB events detected in photon-by-photon mode is about twice that detected with SRM in a single detection layer.

Figure 5 shows the MCAL effective area for different off-axis angles calculated from Monte Carlo simulations. The estimated effective area is about $300 \mathrm{~cm}^{2}$ at $1 \mathrm{MeV}$. Although the effective area increases at higher energies, the limited thickness of

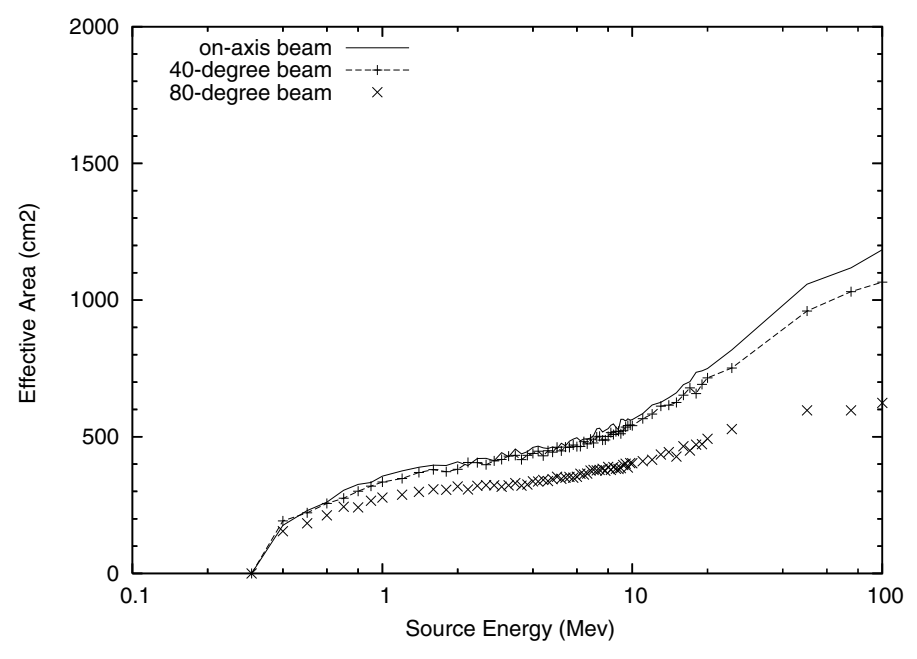

Fig. 5. MCAL total effective area as a function of energy, for different incident angles. Solid line: on-axis beam. Pluses: $40^{\circ}$ off-axis beam. Crosses: $80^{\circ}$ off-axis beam.

the instrument prevents full containment of secondary particles in the pair conversion regime. At low energy, between $330 \mathrm{keV}$ and about $1 \mathrm{MeV}$, the effective area is strongly dependent on the energy threshold of each detector's bar. Moreover the error on energy estimation, based on weighing the signals from both photodiodes for each bar, becomes larger as the energy approaches the threshold, so the low energy response requires careful calibration. Details on the MCAL effective area and energy estimation algorithm are reported in Labanti et al. (2008).

Figure 6 shows the spectral fitting with a power law model for the MCAL data of GRB 080514B, in the energy range 500$5000 \mathrm{keV}$, using preliminary calibration parameters. The solid line represents the best-fit model folded with the MCAL response (reduced $\chi^{2}=1.1$ with 12 degrees of freedom). The photon index is $-2.66_{-0.25}^{+0.30}$, where quoted errors are at the $90 \%$ confidence level. This value is consistent within errors with the high energy photon index obtained fitting Konus-Wind and SuzakuWAM data with a Band model, as reported in Golenetskii et al. (2008e) and Hanabata et al. (2008). As observed by MCAL, the burst fluence in the $500-5000 \mathrm{keV}$ energy range is $(7.8 \pm$ $1.5) \times 10^{-6} \mathrm{erg} / \mathrm{cm}^{2}$ which is about $60 \%$ of the value expected integrating the Konus-Wind spectra in the same energy range. Below $500 \mathrm{keV}$ the number of observed events is lower than expectations and an acceptable fit cannot be obtained, at the moment. This could be due to an overestimation of the effective area at low energy. To validate the effective area using in-flight data, a cross calibration activity is currently ongoing using bright GRBs detected both by MCAL and Konus-Wind. The low energy effective area assessment, in order to properly estimate the peak energy for detected bursts, as well as the total fluence discrepancy are the most important items to be addressed by the cross calibration activity. Due to the fact that the on-board trigger logic has been steadily active since February 2008, at the time of writing only a few GRB events are suitable for this activity, which is currently in progress.

\subsection{Timing performance}

The MCAL high time resolution capability is one of the remarkable characteristics of the instrument, and can be clearly inferred 
GRB 080514B

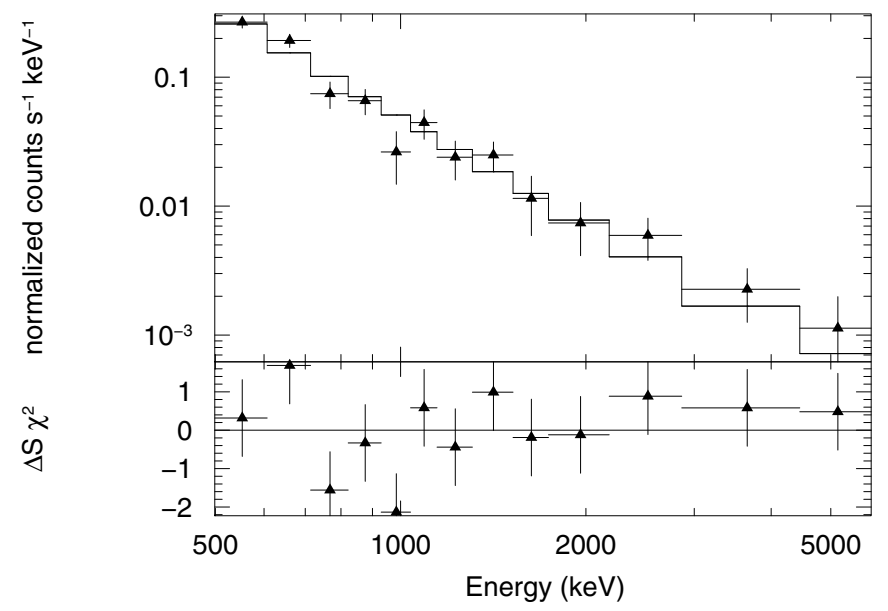

Fig. 6. Spectral fitting of GRB 080514B. Filled triangles: MCAL data. Solid line: best fit power law model.

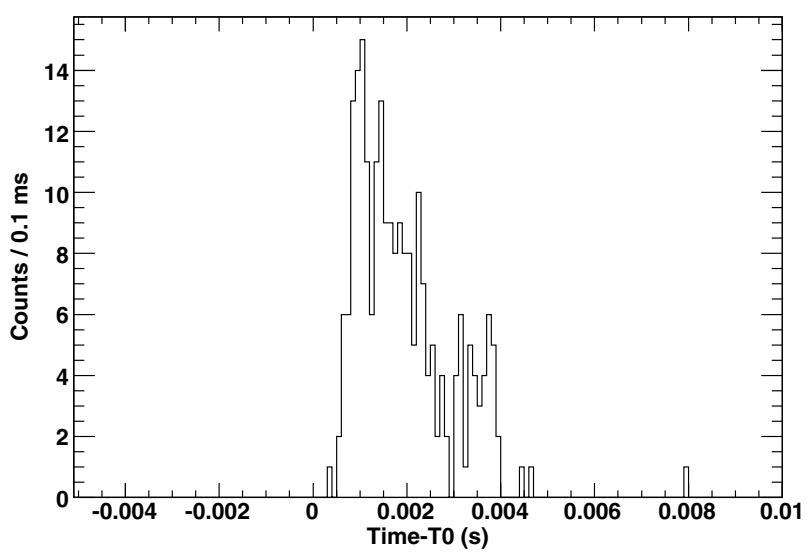

Fig. 7. Light curve of the event triggered on 2008-03-23 at 02:28:25 UT.

from the light curve reported in Fig. 7. This event was triggered on-board on 2008-03-23 at 02:28:25 UT in the $64 \mathrm{~ms}$ time window. It has a duration of about $4 \mathrm{~ms}$ and it is bright enough to be binned with $100 \mu$ s time bins. Since the activation of the onboard trigger logic, several events like this one have been detected (Fuschino et al. 2008b). Despite the terrestrial origin of these gamma-ray flashes being considered the most probable explanation (Fishman et al. 1994; Smith et al. 2005), their possible cosmic origin is under investigation as well.

\section{Conclusions}

MCAL is tailored to the detection of medium-bright GRBs with peak energies above a few hundred $\mathrm{keV}$, as expected from sensitivity calculations and experimental evidence reported in Sect. 3.2. The main characteristics of the instrument are its spectroscopic capabilities in the $\mathrm{MeV}$ range and the microsecond timing accuracy. Between 22nd June 2007 and 30th June 2008 MCAL detected 51 GRBs, with an average detection rate of about $1 \mathrm{GRB} /$ week. A preliminary flux calibration is in good agreement with expectations. Since the beginning of February
2008 the on-board trigger logic has been active and calibration activities are in progress.

Acknowledgements. We wish to thank Kevin Hurley and Valentin Pal'shin for fruitful discussion and IPN support. AGILE is a mission of the Italian Space Agency, with co-participation of INAF (Istituto Nazionale di Astrofisica) and INFN (Istituto Nazionale di Fisica Nucleare).

\section{References}

Aptekar, R. L., Frederiks, D. D., Golenetskii, S. V., et al. 1995, Space Sci. Rev., 71,265

Band, D., et al. 1993, ApJ, 413, 281

Band, D. L. 2003, ApJ, 588, 945

Barthelmy, S. D. 2000, Proc. SPIE, 4140, 50

Cocco, V., Longo, F., \& Tavani, M. 2002, Nucl. Instr. Meth. A, 486, 623

Del Monte, E., Costa, E., Di Persio, G., et al. 2007, Proc. of SciNeGHE 2007, Frascati Physics Series, 45, 201, [arXiv: 0712 . 1548]

Del Monte, E., Feroci, M., Pacciani, L., et al. 2008, A\&A, 478, L5,

Endo, A., Tashiro, M., Urata, Y., et al. 2007, GRB Coordinates Network, 6672

Feroci, M., Costa, E., Del Monte, E., et al. 2007a, GRB Coordinates Network, 6668

Feroci, M., Costa, E., Soffitta, P., et al. 2007b, Nucl. Instr. Meth. A, 581, 728

Fishman, C. J., et al. 1994, Science, 264, 1313

Fuschino, F., Labanti, C., Galli, M., et al. 2008a, Nucl. Instr. Meth. A, 588, 17

Fuschino, F., et al. 2008b, in preparation

Ghirlanda, G., Galli, M., Longo, F., et al. 2004, AIP Conf. Proc., 727, 704

Giuliani, A., et al. 2008a, A\&A, accepted [arXiv: 0809.1230]

Giuliani, A., et al. 2008b, GRB Coordinates Network, 7716

Golenetskii, S., Aptekar, R., Mazets, E., et al. 2007a, GRB Coordinates Network, 7137

Golenetskii, S., Aptekar, R., Mazets, E., et al. 2007b, GRB Coordinates Network, 6671

Golenetskii, S., Aptekar, R., Mazets, E., et al. 2007c, GRB Coordinates Network, 6879

Golenetskii, S., Aptekar, R., Mazets, E., et al. 2007d, GRB Coordinates Network, 7155

Golenetskii, S., Aptekar, R., Mazets, E., et al. 2008a, GRB Coordinates Network, 7219

Golenetskii, S., Aptekar, R., Mazets, E., et al. 2008b, GRB Coordinates Network, 7263

Golenetskii, S., Aptekar, R., Mazets, E., et al. 2008c, GRB Coordinates Network, 7487

Golenetskii, S., Aptekar, R., Mazets, E., et al. 2008d, GRB Coordinates Network, 7548

Golenetskii, S., Aptekar, R., Mazets, E., et al. 2008e, GRB Coordinates Network, 7751

Hanabata, Y., Uehara, T., Fukazawa, Y., et al. 2008, GRB Coordinates Network, 7752

Holland, S. T., Barthelmy, S. D., Baumgartner, W. H., et al. 2007, GRB Coordinates Network, 6949

Hurley, K. 2008, private communication

Kira, C., Ohno, M., Uehara, T., et al. 2007a, GRB Coordinates Network, 6634

Kira, C., Ohno, M., Uehara, T., et al. 2007b, GRB Coordinates Network, 6616

Kira, C., Ohno, M., Uehara, T., et al. 2007c, GRB Coordinates Network, 6931

Kodaka, N., Tashiro, M., Terada, Y., et al. 2008, GRB Coordinates Network, 7551

Labanti, C., et al. 2006, Proc. SPIE, 6266, 62663

Labanti, C., Marisaldi, M., Fuschino, F., et al. 2008, Nucl. Instr. Meth. A, accepted [arXiv: 0810.1842]

Longo, F., Cocco, V., \& Tavani, M. 2002, Nucl. Instr. Meth. A, 486, 610

Marisaldi, M., Labanti, C., Fuschino, F., et al. 2008, Proc. of Gamma Ray Bursts 2007, November 5-9, Santa Fe, NM, AIP Conf. Proc., 1000, 531

Meegan, C., Bhat, N., Connaughton, V., et al. 2007, AIP Conf. Proc., 921, 13

Michelson, P. F. 2007, in American Institute of Physics Conference Series, The First GLAST Symposium, ed. S. Ritz, P. Michelson, \& C. A. Meegan, 921, 8 Ohno, M., Uehara, T., Takahashi, T., et al. 2007, GRB Coordinates Network, 7141

Onda, K., Tashiro, M., Terada, Y., et al. 2008a, GRB Coordinates Network, 7158 Onda, K., Tashiro, M., Terada, Y., et al. 2008b, GRB Coordinates Network, 7508 Pal'shin, V., \& Hurley, K. 2007, private communication

Prest, M., et al. 2003, Nucl. Instr. Meth. A, 501, 280

Rapisarda, M., et al. 2008, GRB Coordinates Network, 7715

Smith, D. M., et al. 2005, Science, 307, 1085

Tavani, M., Barbiellini, G., Argan, A., et al. 2008a, A\&A, submitted

Tavani, M., et al. 2008b, Nucl. Instr. Meth. A, 588, 52

Terada, Y., Onda, K., Tashiro, M., et al. 2008, GRB Coordinates Network, 7326 
Ubertini, P., Lebrun, F., Di Cocco, G., et al. 2003, A\&A, 411, L131

Uehara, T., Ohno, M., Takahashi, T., et al. 2008, GRB Coordinates Network, 7218

Wigger, C., Hajdas, W., Smith, D. M., et al. 2004, Nucl. Phys. B Proc. Suppl., 132,331

Yamaoka, K., Sugita, S., Ohno, M., et al. 2006, Proc. SPIE, 6266, 153

${ }^{1}$ INAF - IASF Bologna, via Gobetti 101, 40129 Bologna, Italy e-mail: marisaldi@iasfbo.inaf.it

2 ENEA, via Martiri di Monte Sole 4, 40129 Bologna, Italy

3 INAF - IASF Roma, via del Fosso del Cavaliere 100, 00133 Roma, Italy

4 Dipartimento di Fisica Università di Trieste, via A. Valerio 2, 34127 Trieste, Italy

5 INFN Trieste, via A. Valerio 2, 34127 Trieste, Italy

${ }^{6}$ INFN Pavia, via Bassi 6, 27100 Pavia, Italy

7 Dipartimento di Fisica Nucleare e Teorica, Università di Pavia, via Bassi 6, 27100 Pavia, Italy

8 INAF - IASF Milano, via E. Bassini 15, 20133 Milano, Italy

9 CIFS Torino, via le Settimio Severo 63, 10133 Torino, Italy
10 Dipartimento di Fisica, Università Tor Vergata, via della Ricerca Scientifica 1, 00133 Roma, Italy

11 Dipartimento di Fisica, Università di Torino, Torino, Italy

12 Dipartimento di Fisica, Università dell'Insubria, via Valleggio 11, 22100 Como, Italy

13 INFN Milano-Bicocca, Piazza della Scienza 3, 20126 Milano, Italy

${ }_{14}$ IKI, Moscow, Russia

15 INFN Roma "La Sapienza", p.le Aldo Moro 2, 00185 Roma, Italy

16 Dipartimento di Fisica, Università La Sapienza, p.le Aldo Moro

2, 00185 Roma, Italy

17 CNR-IMIP, Area della Ricerca di Montelibretti, Roma, Italy

18 INFN Roma “Tor Vergata”, via della Ricerca Scientifica 1, 00133 Roma, Italy

19 ENEA Frascati, via Enrico Fermi 45, 00044 Frascati, Roma, Italy

20 ASI Science Data Center, via E. Fermi 45, 00044 Frascati, Roma, Italy

${ }_{21}$ Agenzia Spaziale Italiana, viale Liegi 26, 00198 Roma, Italy 